\title{
Secular change in dental development in New Mexican females
}

Anna L.M. Rautman ${ }^{1}$, Heather J.H. Edgar ${ }^{1}$

${ }^{1}$ Department of Anthropology and Maxwell Museum of Anthropology, University of New Mexico Albuquerque, New Mexico 87131

Keywords: Dental Development; Hazards Analysis; Secular Change

ABSTRACT Recent research has indicated a dramatic acceleration of dental development in $20^{\text {th }}$ century European Americans in Tennessee and Arizona, resulting in developmental stages being reached at earlier calendar ages. In order to determine whether this rate change is also observed in New Mexico, radiographs from two cohorts of European American female orthodontic patients with known ages were used to compare age by stage of development. The cohorts date to the 1970's (n=101) and the 1990's (n=93) and were between 5-11 years of age. Dental developmental stages were recorded for five mandibular teeth.

The average calendar age difference between

Dental development is generally thought to be a precise method for estimating an individual's chronological age during growth, because it seems to be less affected by environmental variation than long bone length. However, secular change has been documented in the timing of dental development (Nadler, 1998; Cardoso et al., 2010; O'Neill, 2012; Sasso et al., 2012). Secular change refers to non-genetic, directional changes in the timing, rate, and magnitude of development over successive generations, often related to environmental factors (Garn, 1987; O'Neill, 2012). Evidence of secular change has been reported across numerous populations and in many body systems, including height and age of menarche (Cole, 2000; Thompson et al., 2002; Cardoso et al., 2010).

Previous research has shown that children in the United States and Europe are reaching stages of dental development at younger ages then had previous generations (Nadler, 1998; Cardoso et al., 2010; O'Neill, 2012; Sasso et al., 2012). Nadler (1998) noted that patients in Tucson, Arizona in the 1990's who were described as Caucasian and between 8.5-14.5 years were reaching stages of dental development at younger chronological ages than similar patients had in the 1970's. Specifically, he detected a reduction of 1.52 years in the obtainment of dental development stage G (Demirjian et al., 1973) of the mandibular canine in cohorts per tooth and developmental stage combination was less than one month, but varies among tooth/stage combinations by up to 13 months. A Pearson's chi square test found no significant difference between the two cohorts for the 22 tooth/ stage combinations. However, Cox Hazards Analysis demonstrated significant differences between the cohorts for five of the 22 tooth and stage combinations. Contrary to previous findings, the calendar age of the 1990's cohort is older for 16 of the 22 tooth/stage combinations than the 1970's cohort. This runs counter to the general trend of acceleration in development observed in multiple systems.

females between two cohorts, 1972-1974 and 19921994. Work by O'Neill (2012) also showed an increase in the rate of dental development in patients described as American white from Memphis, Tennessee. This study examined the dental development of all mandibular teeth and found a 1.1-year reduction in chronological age relative to dental age between two cohorts from 1980-1985 and 2005-2010.

Research in Europe has also demonstrated a reduction in age of dental development stage attainment. In Portugal, modern girls were shown to have matured dentally 1.47 years faster than girls from half a century ago (Cardoso et al., 2010). The historic sample was comprised of skeletons of individuals who died between 1903 and 1972, with the majority of the deaths occurring between 1920 and 1950. The modern sample was comprised of dental patients whose radiographs were taken between 1998 and 2006. For both samples, the first seven mandibular teeth were examined. A study in Croatia of seven left mandibular teeth observed an acceleration of 0.83 years in girls' rate of dental development between 1977-1979 and 2007-2009 (Sasso et al., 2012).

\footnotetext{
Correspondence to: Heather Edgar

Department of Anthropology and Maxwell Museum of Anthropology, University of New Mexico, Albuquerque, New Mexico 87131

Hjhedgar@unm.edu
} 
Given an increase in the rate of dental development observed for Europeans and European Americans, this study examines whether there is evidence for secular change in the timing of dental development in European American females in Albuquerque, New Mexico. The hypothesis was that more recent patients obtained stages of dental development at younger ages than had patients in an earlier cohort.

\section{MATERIALS AND METHODS}

The sample consists of 194 radiographs in two cohorts of female patients of European-American ancestry (Edgar et al., 2011) who were less than 11 years old. One orthodontist in Albuquerque, New Mexico saw all the patients. Cohort one included 101 radiographs of patients who were seen between 1973 and 1979. Cohort two included 93 radiographs of patients seen between 1990-1999. While the patients were living in New Mexico at the time of their treatment, how long they had lived in New Mexico was not known.

Through observations of panoramic oral radiographs, one author (ALMR) assigned a dental development stage to every tooth, maxillary and mandibular, deciduous and permanent, using a 13 stage method commonly used in studies of dental development (hereafter referred to as "the Moorrees method") (Moorrees et al., 1963 a,b; AlQahtani et al., 2010). Because of limited observations, only five teeth, all mandibular, are included in this analysis: the canine, both premolars, and the second and third molars. Although direct scoring of the radiographs was completed using the Moorrees method for dental development stages, scores were converted to stages described by Demirjian (1973, hereafter referred to as "the Demirjian method") so that results from the New Mexico sample could be compared to those reached by Nadler (1998) from Tucson, Arizona and O'Neill (2012) in Memphis, Tennessee. The conversion used the written descriptions of the progress of dental development to match descriptions in the two methods. Details of the conversion are shown in Figure 1.

An intra-observer error test was run on a subset of 40 radiographs, 20 from each cohort. The consistency between the two sets of observations was tested using weighted and unweighted Cohen's Kappa tests (Cohen, 1960; Viera and Garrett, 2005). The mean and median age and standard deviation

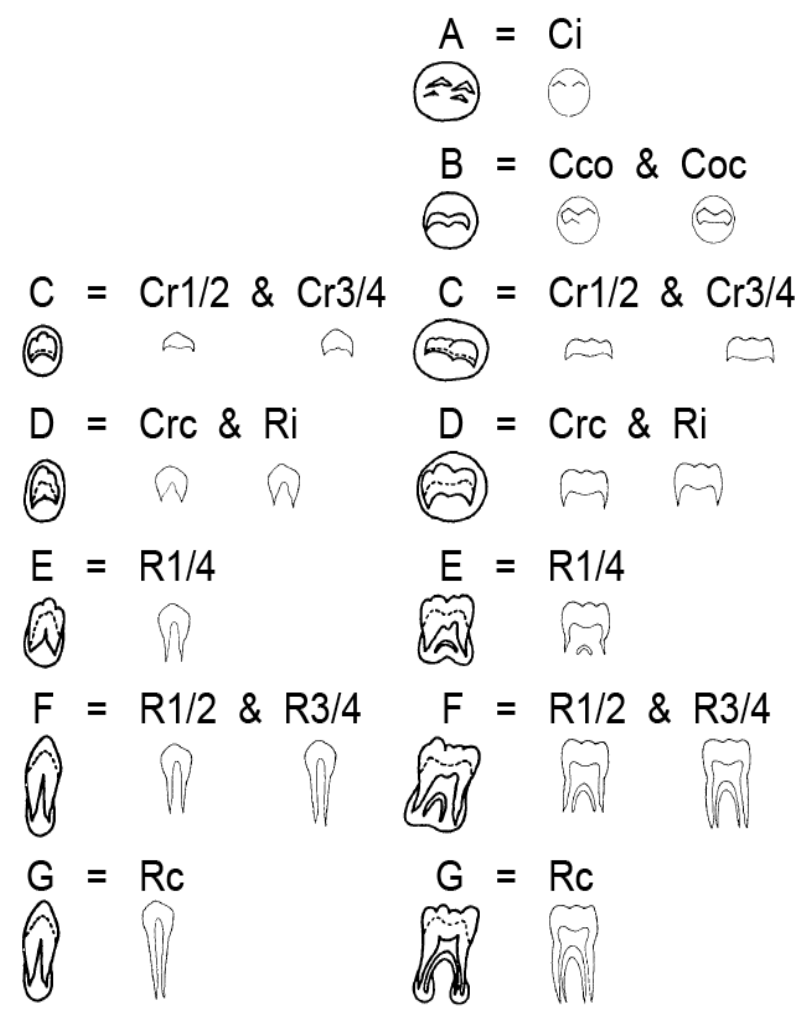

Fig. 1. Conversion between the Demirjian method $(A-G)$ and the Moorree's method (Ci - Rc) dental development stages. Anterior teeth are pictured on the left, posterior teeth on the right.

were calculated for each developmental stage per tooth for each cohort. Using the mean ages, Pearson's chi square was used to test for significant differences for each stage per tooth between the cohorts (Gotelli and Ellison, 2004). Cox Proportional Hazards Analysis (Cox and Oakes, 1984; Fox, 2002) was used to analyze individual age differences in survivorship of each stage. In this analysis, the event of interest is the transition to the next development stage. Individual ages represent the time observation. This allows for analysis of relative ages and frequency of individuals who survive to the stage.

\section{RESULTS}

\section{Intraobserver Test}

The weighted kappa score testing intraobserver error for observations of the five mandibular teeth included in this analysis is 0.917 , and the unweighted kappa score is 0.676. Both kappa scores demonstrate agreement between observa- 
tion, "almost perfect agreement" and "substantial agreement," respectively (Viera and Garrett, 2005). Given the ordinal nature of the development stages, the weighted kappa is more applicable.

\section{Dental Development in New Mexico: The Moorrees Method}

The mean age of dental development stages across all five teeth is younger in the 1970's cohort than in the 1990's cohort for the majority of stages. This indicates a slowing of dental development. This difference was usually small, with a mean absolute difference of 3.3 months. However, there are directional differences in which cohort is older for any given tooth/stage combination. Because sometimes the 1970's cohort is older for a given stage of development, and sometimes the 1990's cohort is older, adding all differences between the cohorts together results in the 1990's cohort being on average only 0.2 months older.

Table 1 presents the sample size and frequency per tooth/stage combinations, as well as Pearson's chi square and Cox Hazards Analysis results. Only two tooth/stage combination differences between cohorts were greater than six months: the canine at root one half (10 months) and the crown complete stage in the fourth premolar (13 months). Of the 22 tooth/stage combinations, only six had measurable differences between mean ages older in the 1970's cohort than in the 1990 's cohort. Three of these tooth/stage combinations were in the second molar (crown threequarters, crown complete and root one-half). The other tooth/stage combinations seen at older ages in the 1970's cohort were the canine (root complete), third premolar (root complete), and the fourth premolar (root one-quarter). Of these six tooth/stage combinations, only the fourth premolar (root one-quarter) and second molar (crown three-quarter) had differences greater than one month, 3.81 months and 1.05 months, respectively.

Pearson's chi square and Cox Hazards Analysis disagree about significant differences between the cohorts. No Pearson's chi square result indicates significant differences in the mean or median ages between the two cohorts. This is true for all tooth/stage combinations, and regardless of whether the 1970's or 1990's cohorts showed any particular tooth/stage combination at an earlier age. In contrast, Cox Hazards Analysis detects significant differences between cohorts $(p<0.05)$ in the survivorship of development stages as patients develop out of a given dental stage for five of the 22 tooth/stage combinations. Timing of the canine (root one-half and root three-quarter), both premolars (third: root one-quarter; fourth: root initialized), and the second molar (root initialized) is significantly different between cohorts. Of these five tooth/stage combinations, the mean difference was 5.4 months. The mean age of the 1990's cohort was always older than the mean age of the 1970's cohort.

\section{Dental Development in New Mexico: The Demirjian Method}

After conversion of the observed Moorrees method dental development stages to the Demirjian method stages, the difference in mean ages between the two cohorts remains, with the 1990's cohort mean being slightly older. The absolute mean difference is 3.01 months. When directional differences between the two cohorts were considered, the difference is 1.9 months with the 1990's cohort as the older.

Only one tooth/stage combination difference is greater than six months (canine, stage F). Of the 17 tooth/stage combinations considered, only five (canine, stage G; third premolar, stage G; fourth premolar, stage $\mathrm{E}$; second molar, stage $\mathrm{F}$; and third molar, stage C) had mean ages such that younger chronological ages were associated with development stages in the 1990's cohort.

The conversion of dental development scores from the Morrees method to the Demirjian method does not result in any significant Pearson's chi square tests of the mean age differences between the cohorts. Cox Hazards Analysis of the Demirjian method stage data demonstrates four of 17 tooth/stage combinations having significant differences in survivorship of stages between cohorts $(p<0.05)$. These four, canine (stage $F)$, third premolar (stages E and F) and fourth premolar (stage D) have a mean age difference of 4.8 months. In all tooth/stage combinations the mean age of survivorship of the 1990's cohort is older than the 1970's cohort. 


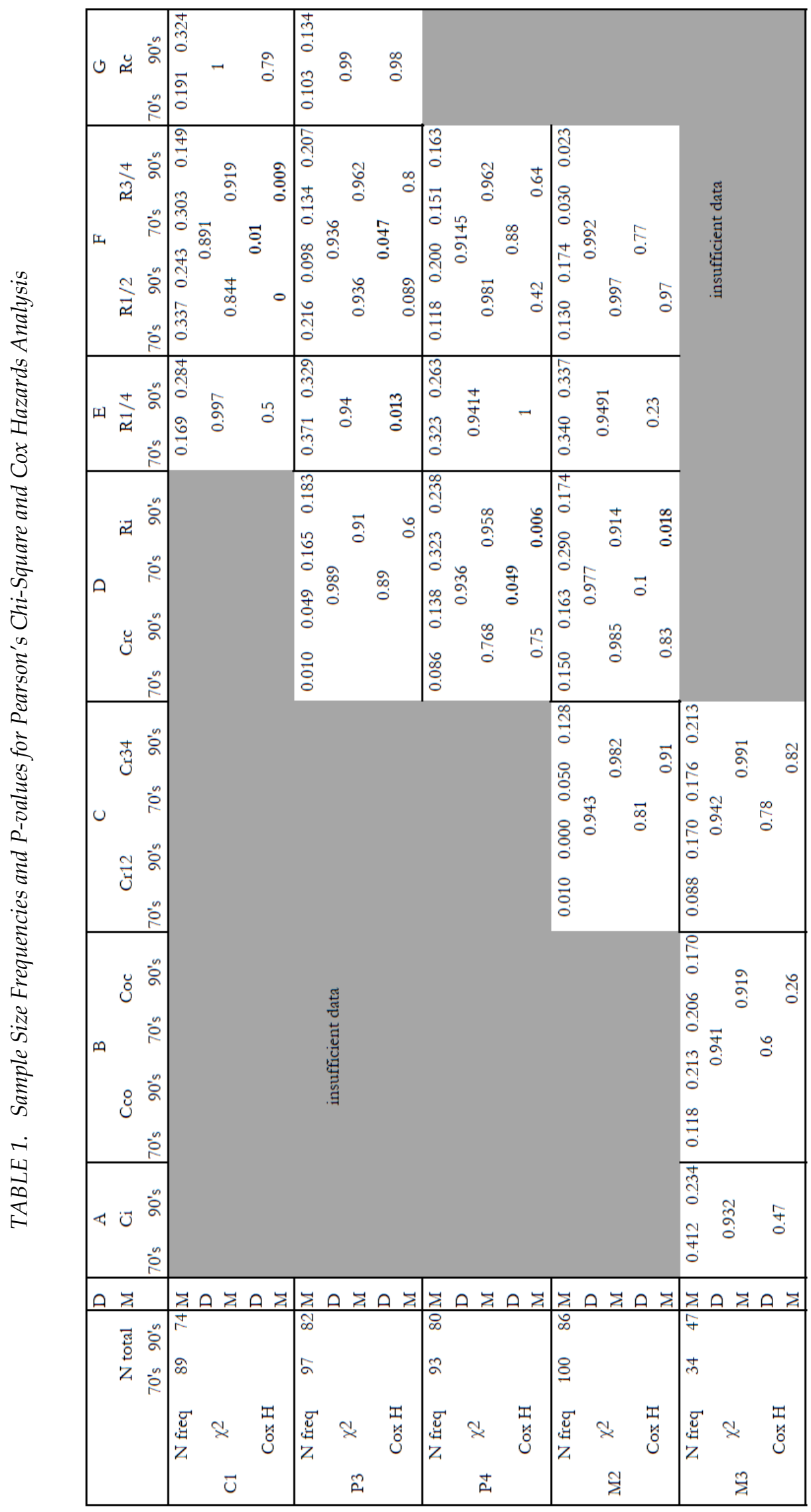




\section{Arizona, New Mexico, and Tennessee Compared}

All tooth/stages are seen at younger chronological ages in New Mexico than in Tennessee (O'Neill, 2012). This difference ranges between 0.7 and 2.52 years, with an average difference of 1.52 years. A similar pattern with less difference between the samples is observed when New Mexico and Arizona are compared (Nadler, 1998).The average difference between the southwest states is 0.69 years, with the Arizona sample older. The range of differences is from the Arizona sample being older by 1.39 years to the New Mexico sample being older by 0.13 years. Figure 2 shows the interquartile range for the New Mexico and Tennessee samples as well as a range of two standard deviations for the Arizona sample, for which interquartile could not be computed.

\section{DISCUSSION}

Considering the evidence for secular change seen by previous authors, it was expected that the 1970 's cohort would have achieved developmental stages at a later average age than 1990's cohort. However, regardless of the method used to measure dental development, Moorrees or Demirjian, it is apparent that in New Mexico, the 1970's cohort achieved dental development stages at younger ages on average than the 1990 cohort. Our results do not agree with the positive secular trend of dental development as observed previously in Arizona, Tennessee, Portugal, and Croatia. Furthermore, the magnitude of difference in age between cohorts was much larger elsewhere, ranging from 0.83 years in Croatia to 1.52 years in Arizona, compared to the average difference of 0.42 years observed in New Mexico.

Within the New Mexico sample, the significant differences in the Cox Hazards Analysis are primarily seen in eight and nine year olds. This observation raises the question of possible external and/or somatic environmental influences of dental development at that time. This age range generally falls between the mid-growth spurt and the adolescent growth spurt associated with puberty (Eveleth and Tanner, 1976; Bailey, 1991; Bogin, 1999). There is a slower period of body growth between early childhood and puberty. During this lull between the mid-growth spurt and the adolescent growth spurt the energy not used in skeletal growth is allocated elsewhere (Hill and Hurtado,
1996). One possible direction for this energy is social learning (Hill and Kaplan, 1999). At the same time, variation between individuals increases in multiple body systems throughout development (Ogden et al., 2002; Lin et al., 2006). Since the period of greatest variability in dental development is correlated with a lull in skeletal growth, it may indicate that energy not being used in rapid skeletal growth is at least in part being diverted to dental development.

While it appears that the mean age of dental development is not changing in New Mexico, the mean ages in Tennessee are getting progressively younger, getting closer to the early mean age already obtained in New Mexico. This is true for the Arizona sample as well, with one tooth/stage exception (canine, stage G). However, there are differences between the studies from Arizona, New Mexico, and Tennessee in time periods from which cohorts were observed, complicating direct comparison. The first cohort in Arizona and New Mexico was taken from 1970's patient records, while patients in the first cohort from Tennessee were seen in the 1980's. The second cohorts were from the 1990's for Arizona and New Mexico and 2000's for Tennessee.

\section{CONCLUSION}

This study finds no evidence of positive secular change of dental development among New Mexican European American females, as had been observed previously in Tennessee and Arizona. In fact, the only significant differences detected show that the more recent New Mexican cohort has less developed teeth at specific chronological ages, exactly opposite of the trend observed by Nadler (1998) and O'Neill (2012).

Causation is often an unexplored issue in studies of secular change. Possible sources of change are external environmental factors such as nutrition, chemical exposure, and disease, as well as somatic environmental effects of energy allocation trade-offs between different body systems (Kieser, 1992; Kieser et al., 1997; Euling et al., 2008; Walker and Hamilton, 2008; Cardoso et al., 2010; Patisaul and Jefferson, 2010). In addition to external and somatic factors, there are genetic factors as well that may contribute to varied rates of dental development. While it is possible that genetic differences are the cause of the observed differences, the fact that all three studies were of European Ameri- 


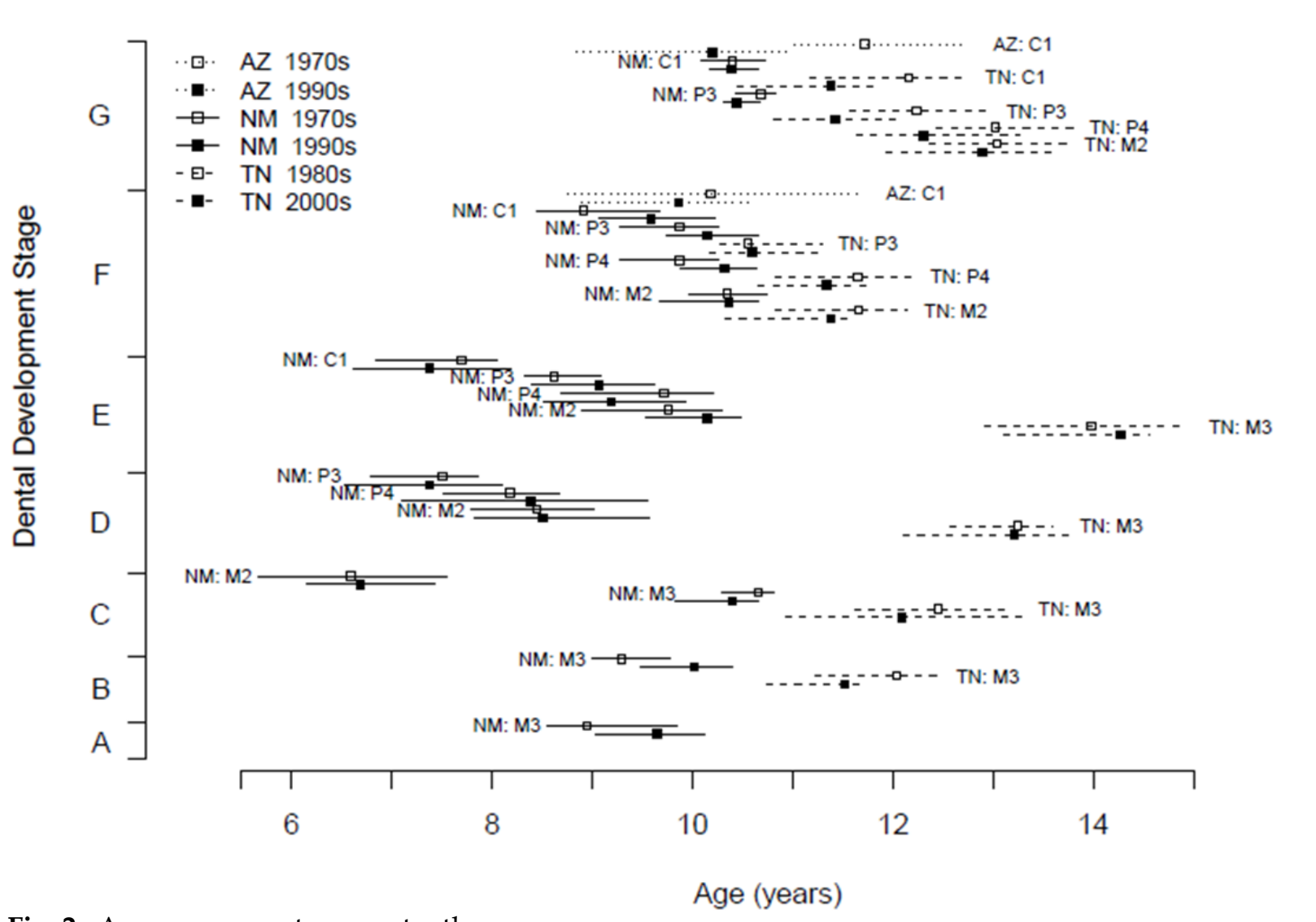

Fig. 2. Age range per stage per tooth.

can females makes this less likely. This would suggest that external and/or somatic environmental factors contribute to the results observed. To address this, a finer scale analysis of when changes in the pace of dental development occur is needed. Such research should consider how the different external, somatic, and genetic factors interact with each other to influence dental development.

\section{LITERATURE CITED}

AlQahtani S, Hector M, and Liversidge H. 2010. Brief communication: The London atlas of human tooth development and eruption. Am J Phys Anthropol 142:481-490.

Bailey RC. 1991. The comparative growth of Efe pygmies and African farmers from birth to age 5 years. Ann Hum Biol 18:113-120.

Bogin B. 1999. Patterns of Human Growth. 2nd Ed. Cambridge, United Kingdom: Cambridge University Press.

Cardoso HFV, Heuze Y, and Julio P. 2010. Secular Change in the Timing of Dental Root Matura tion in Portuguese Boys and Girls. Am J Hum
Biol 2009:245-275.

Cohen J. 1960. A coefficient of agreement for nomi nal scales. Educational and Psychological Measurement 20:37-46.

Cole TJ. 2000. Secular trends in growth. Proc Nutr Soc 59:371-324.

Cox DR, Oakes D. 1984. Analysis of Survival Data. London: Chapman and Hall.

Demirjian A, Goldstein H, Tanner JM. 1973. A New System of Dental Age Assessment. Hum Biol 45:211-227.

Edgar HJH, Daneshvari S, Harris E, and Kroth PJ. 2011. Inter-Observer Agreement on Subjects' Race and Race- Informative Characteristics. PLoS ONE 6:e23986.

Euling SY, Herman-Giddens ME, Lee PA, Selevan SG, Juul A, Sørensen TIA, Dunkel L, Himes JH, Teilmann G, et al. 2008. Examination of US Puberty-Timing Data from 1940 to 1994 for Secular Trends: Panel Findings. Pediatrics 121.

Eveleth PB, Tanner JM. 1976. Worldwide Variation in Growth. Cambridge, United Kingdom: Cambridge University Press. 
Fox J. 2002. Cox Proportional-Hazards Regression for Survival Data. In: An R and S-PLUS Companion to Applied Regression.

Garn SM. 1987. The Secular Trend in Size and Maturation Timing and Its Implications for $\mathrm{Nu}$ tritional Assessment. J Nutr 117:817-823.

Gotelli NJ, Ellison AM. 2004. A Primer of Ecological Statistics. Massachusetts, U.S.A.: Sinauer Associates, Inc.

Hill K, Kaplan H. 1999. Life History Traits in Humans: Theory and Empirical Studies. An nual Rev of Anthropol 28:397-430.

Hill KR, Hurtado AM. 1996. Body Size and the Timing of Sexual Maturity. In: Aché Life History: The Ecology and Demography of a Foraging People. New York: Walter de Gruyter, Inc. p 341-373.

Kieser JA, Groeneveld HT, Da Silva PCF. 1997. Dental Asymmetry, Maternal Obesity, and Smoking. Am J Phys Anthropol 102:133139.

Kieser JA. 1992. Fluctuating odontometric asym metry and maternal alcohol consumption. Ann Hum Biol 19:513-520.

Lin N-H, Ranjitkar S, MacDonald R, Hughes TE, Taylor JA, Townsend GC. 2006. New growth references for assessment of stature and skeletal maturation in Australians. Aust Orthod J 22:1-10.

Moorrees CFA, Fanning EA, and Edward E. Hunt Jr. 1963a. Age Variation of Formation Stages for Ten Permanent Teeth. J Den Res 42:1490-1502.
Moorrees CFA, Fanning EA, and Hunt EEJ. 1963b. Formation and Resorption of Three Deciduous Teeth in Children. Am J Phys Anthropol 21:205213.

Nadler GL. 1998. Earlier dental maturation: Fact or fiction? Angle Orthod 68:535-538.

O'Neill KM. 2012. A Secular Increase in the Tempos of Tooth Formation 1980-2010.

Ogden CL, Kuczmarski RJ, Flegal KM, Mei Z, Guo

S, Wei R, Grummer-Strawn LM, Curtin LR, Roch

$\mathrm{AF}$, et al. 2002. Centers for Disease Control and Prevention 2000 Growth Charts for the United States: Improvements to the $1977 \mathrm{Na}$ tional Center for Health Statistics Version. Pediatrics 109:45-60.

Patisaul HB, Jefferson W. 2010. The pros and cons of phytoestrogens. Front Neuroendocri nol 31:400-419.

Sasso A, Špalj S, Mady Maričić B, Sasso A, Ćabov T, Legović M. 2012. Secular trend in the develop ment of permanent teeth in a population of Istria and the Littoral Region of Croatia. J Forensic Sci 57:1-5.

Thompson AM, Baxter-Jones ADG, Mirwald RL, Bailey DA. 2002. Secular Trend in the Development of Fatness During Childhood and Adolescence. Am J Hum Biol 14:669-679.

Viera AJ, Garrett JM. 2005. Understanding Interobserver Agreement: The Kappa Statistic. Family Medicine 37:360-363.

Walker RS, and Hamilton MJ. 2008. Life-History Consequences of Density Dependence and the Evolution of Human Body Size. Curr An thropol 49:115-122. 